\title{
STATISTICAL ANALYSIS OF RECENT FAULT-PLANE SOLUTIONS OF EARTHQUAKES
}

\author{
By A. E. SchEIDEGGER
}

\begin{abstract}
The large number of fault-plane solutions at present available in the literature permit one to calculate several statistical averages that have an important bearing upon geotectonics. The present paper represents a continuation of earlier work in this direction: 101 new fault-plane solutions are listed and the ratio of pressure to tension, strike slip to dip slip, and the average slip angle have been calculated for nine earthquake areas. Some of the older results are thereby corroborated, viz., that the "normal" character of earthquakes is to represent strike-slip faulting, and that the central Asian regions constitute an exception to this rule. In addition, it is now possible to make a breakdown with regard to depth. In this, a peculiar situation is found at $0.03 \mathrm{R}$ depth, where the slip angle reaches a maximum. If the relationship between shallow and deep earthquakes be considered for any one area, however, it turns out that they are on the whole of the same character. Thus, whatever it is that causes earthquakes, acts in a similar fashion at all depths in any one area, but differs from one area to another.
\end{abstract}

\section{INTRODUCTION}

ONE BODY of seismological data that has rapidly been accumulating in recent times concerns fault-plane solutions of earthquakes. These fault-plane solutions are concerned with a determination of the position of the fault plane and of the motion direction in the focus of a seismic shock. In general, the fault plane is given by its dip and dip direction, and the motion direction is given by the dip and dip direction of the plane normal to it (the "auxiliary" plane). The nature of the seismological evidence is such that it is often not possible to decide which is the fault plane and which is the auxiliary plane.

If the fault planes are plotted on a map of the region in which a series of earthquakes occurred, it is usually not possible to discern any orderly pattern. It appears that the way in which a particular earthquake occurs is mostly due to chance, and that only certain statistical averages have any significance with regard to questions of tectonics. The writer has attempted on a previous occasion to deduce significant statistical averages from the body of fault-plane solutions then available and to draw inferences therefrom with regard to orogenetic processes. The results then obtained were published earlier (Scheidegger, 1957a).

\section{New Evidence}

Since the writer's earlier paper, some 300 new fault-plane solutions have appeared in the literature. The majority of these refer to very small ("local") earthquakes in central Asia that have been studied by various Russian groups in connection with the local tectonics of that area. A number of local central Asian earthquakes had already been included in the earlier investigation of the writer's, but it seems now hardly justifiable to use these small earthquakes in connection with a discussion of large-scale geotectonics.

Manuscript received for publication March 24, 1959. 
TABLE 1

Fault-Plane Solutions

(See text for explanation of lettered columns )

\begin{tabular}{|c|c|c|c|c|c|c|c|c|}
\hline$A$ & B & $\mathrm{C}$ & D & $\mathbf{E}$ & $\mathrm{F}$ & $\mathrm{G}$ & H & I \\
\hline $\begin{array}{l}\text { Nov. } 161911 \\
21: 25\end{array}$ & $\begin{array}{rl}48 & 3 \mathrm{~N} \\
9 & 1 \mathrm{E}\end{array}$ & 000 & $\begin{array}{l}\mathrm{a}^{\prime \prime} \\
\mathrm{b}\end{array}$ & $\begin{array}{lll}\mathrm{S} & 36 & \mathrm{E} \\
\mathrm{S} & 63 & \mathrm{~W}\end{array}$ & $\begin{array}{l}84 \\
10\end{array}$ & $\begin{array}{l}80 \\
11\end{array}$ & $\begin{array}{l}\text { st } \\
\mathrm{dt}\end{array}$ & $\mathrm{H} 57$ \\
\hline June 261924 & $\begin{array}{rl}56 & 3 \mathrm{~S} \\
158 & 0 \mathrm{E}\end{array}$ & 0.01 & $\begin{array}{l}\mathrm{a}^{\prime \prime} \\
\mathrm{b}\end{array}$ & $\begin{array}{lll}\mathrm{S} & 40 & \mathrm{~W} \\
\mathrm{~S} & 67 & \mathrm{E}\end{array}$ & $\begin{array}{l}86 \\
14\end{array}$ & $\begin{array}{l}77 \\
17\end{array}$ & $\begin{array}{l}\mathrm{sp} \\
\mathrm{dp}\end{array}$ & Gi57 \\
\hline $\begin{array}{l}\text { Jan. } 151931 \\
01: 50: 41\end{array}$ & $\begin{array}{ll}16 & 4 \\
96 & 3 \\
\mathrm{~W}\end{array}$ & 000 & $\begin{array}{l}\mathrm{a} \\
\mathrm{b}\end{array}$ & $\begin{array}{lr}\mathrm{N} & 3 \mathrm{~W} \\
\mathrm{~S} & 88 \mathrm{E}\end{array}$ & $\begin{array}{l}79 \\
74\end{array}$ & $\begin{array}{l}17 \\
12\end{array}$ & $\begin{array}{l}\mathrm{dp} \\
\mathrm{sp}\end{array}$ & M57 \\
\hline $\begin{array}{l}\text { Nov. } 131932 \\
04: 46: 45\end{array}$ & $\begin{array}{rl}43 & 4 \mathrm{~N} \\
137 & 0 \mathrm{E}\end{array}$ & 005 & $\begin{array}{l}\mathrm{a} \\
\mathrm{b}\end{array}$ & $\stackrel{\mathrm{S}}{\mathrm{N}} \underset{58}{8 \mathrm{~W}} \mathrm{~W}$ & $\begin{array}{l}43 \\
70\end{array}$ & $\begin{array}{l}31 \\
51\end{array}$ & $\begin{array}{l}\mathrm{dp} \\
\mathrm{sp}\end{array}$ & M57 \\
\hline $\begin{array}{l}\text { June } 241933 \\
21: 54: 38\end{array}$ & $\begin{array}{r}50 \mathrm{~S} \\
104.2 \mathrm{E}\end{array}$ & 000 & $\begin{array}{l}\mathrm{a} \\
\mathrm{b}\end{array}$ & $\begin{array}{lrl}\mathrm{N} & 0 & \mathrm{E} \\
\mathrm{S} & 54 & \mathrm{E}\end{array}$ & $\begin{array}{l}70 \\
35\end{array}$ & $\begin{array}{l}62 \\
41\end{array}$ & $\begin{array}{l}\mathrm{sp} \\
\mathrm{dp}\end{array}$ & V57 \\
\hline $\begin{array}{l}\text { June } 291934 \\
08: 25: 17\end{array}$ & $\begin{array}{r}68 \mathrm{~S} \\
1238 \mathrm{E}\end{array}$ & 011 & $\begin{array}{l}\mathrm{a}^{\prime \prime} \\
\mathrm{b}\end{array}$ & $\begin{array}{lll}\mathrm{S} & 82 & \mathrm{~W} \\
\mathrm{~S} & 02 & \mathrm{~W}\end{array}$ & $\begin{array}{l}45 \\
84\end{array}$ & $\begin{array}{l}12 \\
46\end{array}$ & $\begin{array}{l}\mathrm{dp} \\
\mathrm{sp}\end{array}$ & $\mathrm{R} 57$ \\
\hline $\begin{array}{l}\text { July } 181934 \\
01: 36: 29\end{array}$ & 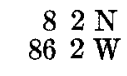 & 0.00 & $\begin{array}{l}\mathrm{a} \\
\mathrm{b}\end{array}$ & $\begin{array}{lll}\mathrm{N} & 90 & \mathrm{E} \\
\mathrm{N} & 11 & \mathrm{~W}\end{array}$ & $\begin{array}{l}63 \\
84\end{array}$ & $\begin{array}{l}13 \\
29\end{array}$ & $\begin{array}{l}\mathrm{dt} \\
\text { st }\end{array}$ & M57 \\
\hline $\begin{array}{l}\text { Sept. } 111935 \\
14: 04: 06\end{array}$ & $\begin{array}{r}43.6 \mathrm{~N} \\
1460 \mathrm{E}\end{array}$ & 000 & $\begin{array}{l}\mathrm{a} \\
\mathrm{b}\end{array}$ & $\begin{array}{lll}\mathrm{N} & 63 & \mathrm{E} \\
\mathrm{S} & 63 & \mathrm{~W}\end{array}$ & $\begin{array}{l}50 \\
40\end{array}$ & $\begin{array}{l}90 \\
90\end{array}$ & $\begin{array}{l}-p \\
-p\end{array}$ & M57 \\
\hline $\begin{array}{l}\text { May } 81936 \\
09: 11: 34\end{array}$ & $\begin{array}{r}58 \mathrm{~S} \\
112.8 \mathrm{E}\end{array}$ & 009 & $\stackrel{a}{b^{\prime \prime}}$ & $\begin{array}{lll}N & 18 & \mathrm{E} \\
\mathrm{S} & 18 & \mathrm{~W}\end{array}$ & $\begin{array}{l}33 \\
57\end{array}$ & $\begin{array}{l}90 \\
90\end{array}$ & $\begin{array}{l}-t \\
-t\end{array}$ & $\mathrm{R} 57$ \\
\hline $\begin{array}{l}\text { June } 301936 \\
15: 06: 44\end{array}$ & $\begin{array}{r}510 \mathrm{~N} \\
161.1 \mathrm{E}\end{array}$ & 000 & $\begin{array}{l}\mathrm{a} \\
\mathrm{b}\end{array}$ & $\begin{array}{lll}\mathrm{N} & 35 & \mathrm{~W} \\
\mathrm{~S} & 35 & \mathrm{E}\end{array}$ & $\begin{array}{l}66 \\
24\end{array}$ & $\begin{array}{l}90 \\
90\end{array}$ & $\begin{array}{l}-p \\
-p\end{array}$ & M57 \\
\hline $\begin{array}{l}\text { Nov. } 21936 \\
20: 45: 56\end{array}$ & $\begin{array}{r}38.3 \mathrm{~N} \\
141.9 \mathrm{E}\end{array}$ & 000 & $\begin{array}{l}\mathrm{a} \\
\mathrm{b}\end{array}$ & $\begin{array}{lll}\mathrm{N} & 53 & \mathrm{~W} \\
\mathrm{~S} & 53 & \mathrm{E}\end{array}$ & $\begin{array}{l}15 \\
75\end{array}$ & $\begin{array}{l}90 \\
90\end{array}$ & $\begin{array}{l}-\mathrm{p} \\
-\mathrm{p}\end{array}$ & M57 \\
\hline $\begin{array}{l}\text { Aug. } 111937 \\
00: 55: 22\end{array}$ & $\begin{array}{r}65 \mathrm{~S} \\
1165 \mathrm{E}\end{array}$ & 0.09 & $a^{\mathrm{b}^{\prime \prime}}$ & $\begin{array}{lll}\mathrm{S} & 13 & \mathrm{E} \\
\mathrm{N} & 11 & \mathrm{E}\end{array}$ & $\begin{array}{l}24 \\
68\end{array}$ & $\begin{array}{l}68 \\
80\end{array}$ & $\begin{array}{l}\mathrm{dt} \\
\text { st }\end{array}$ & $\mathrm{R} 57$ \\
\hline $\begin{array}{l}\text { May } 231938 \\
07: 18: 28\end{array}$ & $\begin{array}{r}365 \mathrm{~N} \\
1416 \mathrm{E}\end{array}$ & 000 & $\begin{array}{l}a \\
b\end{array}$ & $\begin{array}{lll}\mathrm{N} & 59 & \mathrm{E} \\
\mathrm{S} & 59 & \mathrm{~W}\end{array}$ & $\begin{array}{r}84 \\
6\end{array}$ & $\begin{array}{l}90 \\
90\end{array}$ & $\begin{array}{l}-p \\
-p\end{array}$ & M57 \\
\hline $\begin{array}{l}\text { Aug. } 181938 \\
09: 30: 04\end{array}$ & $\begin{array}{r}3.8 \mathrm{~S} \\
1028 \mathrm{E}\end{array}$ & 001 & $\begin{array}{l}\mathrm{a} \\
\mathrm{b}^{\prime \prime}\end{array}$ & $\begin{array}{lll}\mathrm{N} & 30 & \mathrm{~W} \\
\mathrm{~S} & 30 & \mathrm{E}\end{array}$ & $\begin{array}{l}63 \\
27\end{array}$ & $\begin{array}{l}90 \\
90\end{array}$ & $\begin{array}{l}-p \\
-p\end{array}$ & R57 \\
\hline $\begin{array}{l}\text { Oct. } 201938 \\
02: 19: 29\end{array}$ & $\begin{array}{r}9.2 \mathrm{~S} \\
1230 \mathrm{E}\end{array}$ & 001 & $\begin{array}{l}\mathrm{a}^{\prime \prime} \\
\mathrm{b}\end{array}$ & $\begin{array}{lll}\mathrm{N} & 84 & \mathrm{E} \\
\mathrm{N} & 24 & \mathrm{~W}\end{array}$ & $\begin{array}{l}48 \\
71\end{array}$ & $\begin{array}{l}26 \\
45\end{array}$ & $\begin{array}{l}\mathrm{dt} \\
\mathrm{st}\end{array}$ & R57 \\
\hline $\begin{array}{l}\text { Nov. } 151938 \\
21: 00: 16\end{array}$ & $\begin{array}{r}48 \mathrm{~S} \\
98.8 \mathrm{E}\end{array}$ & 000 & $\begin{array}{l}\mathrm{a} \\
\mathrm{b}\end{array}$ & $\begin{array}{l}\mathrm{N} \\
\mathrm{N} \\
52 \mathrm{E}\end{array}$ & $\begin{array}{l}80 \\
90\end{array}$ & $\begin{array}{r}0 \\
10\end{array}$ & $\begin{array}{l}d- \\
s-\end{array}$ & V57 \\
\hline $\begin{array}{l}\text { Nov. } 171938 \\
\text { 03:54:34 }\end{array}$ & $\begin{array}{r}556 \mathrm{~N} \\
157.7 \mathrm{~W}\end{array}$ & 000 & $\begin{array}{l}\mathrm{a} \\
\mathrm{b}\end{array}$ & $\begin{array}{ll}\mathrm{S} & 58 \mathrm{~W} \\
\mathrm{~S} & 43 \mathrm{E}\end{array}$ & $\begin{array}{l}68 \\
68\end{array}$ & $\begin{array}{l}24 \\
24\end{array}$ & $\begin{array}{l}\text { sp } \\
d p\end{array}$ & M57 \\
\hline $\begin{array}{l}\text { June } 181940 \\
13: 52: 33\end{array}$ & $\begin{array}{r}54 \mathrm{~N} \\
1230 \mathrm{E}\end{array}$ & 008 & $\begin{array}{l}a^{\prime \prime} \\
b\end{array}$ & $\begin{array}{ll}\mathrm{N} & 45 \mathrm{~W} \\
\mathrm{~S} & 70 \mathrm{E}\end{array}$ & $\begin{array}{l}43 \\
50\end{array}$ & $\begin{array}{l}71 \\
73\end{array}$ & $\begin{array}{l}\text { st } \\
\mathrm{dt}\end{array}$ & R57 \\
\hline $\begin{array}{l}\text { Sept. } 221940 \\
22: 51: 58\end{array}$ & $\begin{array}{r}75 \mathrm{~N} \\
123.5 \mathrm{E}\end{array}$ & 0.10 & $\begin{array}{l}\mathrm{a}^{\prime \prime} \\
\mathrm{b}\end{array}$ & $\begin{array}{lll}\mathrm{S} & 58 & \mathrm{E} \\
\mathrm{S} & \mathbf{5 5} & \mathrm{W}\end{array}$ & $\begin{array}{l}38 \\
73\end{array}$ & $\begin{array}{l}28 \\
55\end{array}$ & $\begin{array}{l}\text { st } \\
\mathrm{dt}\end{array}$ & $\mathrm{R} 57$ \\
\hline $\begin{array}{l}\text { Jan. } 311941 \\
02: 38: 40\end{array}$ & $\begin{array}{r}6.5 \mathrm{~S} \\
128.5 \mathrm{E}\end{array}$ & 0.03 & $\begin{array}{l}a^{\prime \prime} \\
b\end{array}$ & $\begin{array}{lll}\mathrm{N} & 12 \mathrm{~W} \\
\mathrm{~S} & 70 \mathrm{E}\end{array}$ & $\begin{array}{l}81 \\
17\end{array}$ & $\begin{array}{l}76 \\
33\end{array}$ & $\begin{array}{l}\text { st } \\
\mathrm{dt}\end{array}$ & R57 \\
\hline
\end{tabular}


TABLE 1-Continued

\begin{tabular}{|c|c|c|c|c|c|c|c|c|c|}
\hline$A$ & B & $\mathrm{c}$ & $\mathrm{D}$ & & $\mathrm{E}$ & $F$ & $\mathrm{G}$ & $\mathrm{H}$ & I \\
\hline $\begin{array}{l}\text { Sept. } 171941 \\
06: 48: 04\end{array}$ & $\begin{array}{rl}0 & 1 \mathrm{~N} \\
122 & 7 \mathrm{E}\end{array}$ & 0.03 & $\begin{array}{l}\mathrm{a}^{\prime \prime} \\
\mathrm{b}\end{array}$ & $\stackrel{N}{S}$ & $\begin{array}{l}90 \mathrm{~W} \\
15 \mathrm{E}\end{array}$ & $\begin{array}{l}66 \\
60\end{array}$ & $\begin{array}{l}33 \\
28\end{array}$ & $\begin{array}{l}\mathrm{dt} \\
\text { st }\end{array}$ & R57 \\
\hline $\begin{array}{l}\text { Nov. } 271941 \\
08: 37: 34\end{array}$ & $\begin{array}{r}66 \mathrm{~S} \\
121.1 \mathrm{E}\end{array}$ & 0.07 & $\begin{array}{l}\mathrm{a}^{\prime \prime} \\
\mathrm{b}\end{array}$ & $\underset{\mathrm{S}}{\mathrm{N}}$ & $\begin{array}{l}43 \mathrm{~W} \\
23 \mathrm{~W}\end{array}$ & $\begin{array}{l}73 \\
37\end{array}$ & $\begin{array}{l}57 \\
29\end{array}$ & $\begin{array}{l}\mathrm{sp} \\
\mathrm{dp}\end{array}$ & $\mathrm{R} 57$ \\
\hline $\begin{array}{l}\text { Aug. } 151947 \\
04: 11\end{array}$ & $\begin{array}{lll}44 & 0 & \mathrm{~N} \\
45 & 0 & \mathrm{E}\end{array}$ & 000 & $\begin{array}{l}\mathrm{a}^{\prime \prime} \\
\mathrm{b}\end{array}$ & $\stackrel{\mathrm{S}}{\mathrm{N}}$ & $\begin{array}{l}10 \mathrm{~W} \\
21 \mathrm{~W}\end{array}$ & $\begin{array}{l}74 \\
19\end{array}$ & $\begin{array}{l}80 \\
60\end{array}$ & $\begin{array}{l}\mathrm{dt} \\
\text { st }\end{array}$ & K58 \\
\hline $\begin{array}{l}\text { Aug. } 151947 \\
04: 58\end{array}$ & $\begin{array}{l}44.0 \mathrm{~N} \\
450 \mathrm{E}\end{array}$ & 0.00 & $\begin{array}{l}\mathrm{a}^{\prime \prime} \\
\mathrm{b}\end{array}$ & $\stackrel{\mathrm{S}}{\mathrm{N}}$ & $\begin{array}{l}17 \mathrm{~W} \\
36 \mathrm{~W}\end{array}$ & $\begin{array}{l}72 \\
28\end{array}$ & $\begin{array}{l}68 \\
41\end{array}$ & $\begin{array}{l}\text { st } \\
d t\end{array}$ & K58 \\
\hline $\begin{array}{l}\text { Apr. } 171948 \\
16: 11: 05\end{array}$ & $\begin{array}{r}330 \mathrm{~N} \\
135.5 \mathrm{E}\end{array}$ & 000 & $\begin{array}{l}\mathrm{a} \\
\mathrm{b}\end{array}$ & $\begin{array}{l}\mathrm{S} \\
\mathrm{N}\end{array}$ & $\begin{array}{l}47 \mathrm{E} \\
47 \mathrm{~W}\end{array}$ & $\begin{array}{r}86 \\
4\end{array}$ & $\begin{array}{l}90 \\
90\end{array}$ & $\begin{array}{l}-\mathrm{p} \\
-\mathrm{p}\end{array}$ & M57 \\
\hline Aug. 191948 & 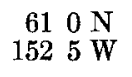 & 001 & $\begin{array}{l}\mathrm{a}^{\prime \prime} \\
\mathrm{b}\end{array}$ & $\stackrel{\mathrm{N}}{\mathrm{N}}$ & $\begin{array}{l}50 \mathrm{~W} \\
65 \mathrm{E}\end{array}$ & $\begin{array}{l}55 \\
50\end{array}$ & $\begin{array}{l}46 \\
42\end{array}$ & $\begin{array}{l}\mathrm{dp} \\
\mathrm{sp}\end{array}$ & G57 \\
\hline Feb. 21949 & $\begin{array}{rl}50 & 5 \mathrm{~N} \\
173 & 5 \mathrm{~W}\end{array}$ & 003 & $a^{\prime \prime}$ & $\begin{array}{l}\mathrm{N} \\
\mathrm{S}\end{array}$ & $\begin{array}{l}05 \mathrm{E} \\
30 \mathrm{~W}\end{array}$ & $\begin{array}{l}62 \\
30\end{array}$ & $\begin{array}{l}78 \\
68\end{array}$ & $\begin{array}{l}\mathrm{sp} \\
\mathrm{dp}\end{array}$ & G57 \\
\hline Apr. 51949 & $\begin{array}{r}41.0 \mathrm{~N} \\
1325 \mathrm{E}\end{array}$ & 0.08 & $a^{\prime \prime}$ & $\stackrel{\mathbf{S}}{\mathbf{S}}$ & $\begin{array}{l}20 \mathrm{~W} \\
75 \mathrm{E}\end{array}$ & $\begin{array}{l}62 \\
82\end{array}$ & $\begin{array}{r}9 \\
28\end{array}$ & $\begin{array}{l}\mathrm{dt} \\
\mathrm{st}\end{array}$ & G57 \\
\hline May 301949 & $\begin{array}{r}165 \mathrm{~N} \\
1460 \mathrm{E}\end{array}$ & 008 & $\begin{array}{l}a^{\prime \prime} \\
b\end{array}$ & $\stackrel{\mathrm{S}}{\mathrm{N}}$ & $\begin{array}{ll}75 & \mathrm{E} \\
75 & \mathrm{~W}\end{array}$ & $\begin{array}{r}86 \\
4\end{array}$ & $\begin{array}{l}90 \\
90\end{array}$ & $\begin{array}{l}-t \\
-t\end{array}$ & G57 \\
\hline Nov. 31949 & $\begin{array}{r}48.5 \mathrm{~N} \\
156.0 \mathrm{E}\end{array}$ & 002 & $\begin{array}{l}\mathrm{a}^{\prime \prime} \\
\mathrm{b}\end{array}$ & $\underset{\mathrm{S}}{\mathrm{N}}$ & $\begin{array}{l}28 \mathrm{E} \\
65 \mathrm{E}\end{array}$ & $\begin{array}{l}85 \\
80\end{array}$ & $\begin{array}{r}10 \\
6\end{array}$ & $\begin{array}{l}\mathrm{dp} \\
\mathrm{sp}\end{array}$ & G57 \\
\hline May 171950 & $\begin{array}{r}39.0 \mathrm{~N} \\
1315 \mathrm{E}\end{array}$ & 0.09 & $\begin{array}{l}\mathrm{a}^{\prime \prime} \\
\mathrm{b}\end{array}$ & $\stackrel{S}{N}$ & $\begin{array}{ll}78 \mathrm{E} \\
78 \mathrm{~W}\end{array}$ & $\begin{array}{l}24 \\
66\end{array}$ & $\begin{array}{l}90 \\
90\end{array}$ & $\begin{array}{l}-t \\
-t\end{array}$ & G57 \\
\hline $\begin{array}{l}\text { Aug } 141950 \\
22: 51: 28\end{array}$ & $\begin{array}{l}27.0 \mathrm{~S} \\
62.5 \mathrm{~W}\end{array}$ & 0.10 & $\begin{array}{l}\mathrm{a} \\
\mathrm{b}\end{array}$ & $\begin{array}{l}\mathrm{N} \\
\mathrm{N}\end{array}$ & $\begin{array}{l}30 \mathrm{E} \\
60 \mathrm{~W}\end{array}$ & $\begin{array}{l}82 \\
77\end{array}$ & $\begin{array}{r}13 \\
8\end{array}$ & $\begin{array}{l}\mathrm{s}- \\
\mathrm{d}-\end{array}$ & DM57 \\
\hline Oct. 251950 & $\begin{array}{r}260 \mathrm{~N} \\
125.0 \mathrm{E}\end{array}$ & 001 & $\begin{array}{l}a^{\prime \prime} \\
b\end{array}$ & $\underset{\mathrm{S}}{\mathrm{N}}$ & $\begin{array}{r}65 \mathrm{E} \\
6 \mathrm{~W}\end{array}$ & $\begin{array}{l}78 \\
30\end{array}$ & $\begin{array}{l}65 \\
34\end{array}$ & $\frac{\mathrm{sp}}{\mathrm{dp}}$ & G57 \\
\hline $\begin{array}{l}\text { Dec. } 101950 \\
\text { 13:23:04 }\end{array}$ & $\begin{array}{r}28 \\
179\end{array}$ & 0.04 & $\stackrel{a}{b}$ & $\stackrel{\mathrm{N}}{\mathrm{S}}$ & $\begin{array}{r}5 \mathrm{~W} \\
83 \mathrm{~W}\end{array}$ & $\begin{array}{l}88 \\
45\end{array}$ & $\begin{array}{r}45 \\
3\end{array}$ & $\mathrm{sp}$ & M57 \\
\hline $\begin{array}{l}\text { Dec. } 121950 \\
19: 51: 49\end{array}$ & $\begin{array}{r}182 \mathrm{~S} \\
167\end{array}$ & 0.00 & $\begin{array}{l}\mathrm{a} \\
\mathrm{b}\end{array}$ & $\stackrel{\mathrm{N}}{\mathrm{S}}$ & $\begin{array}{l}38 \mathrm{~W} \\
48 \mathrm{~W}\end{array}$ & $\begin{array}{l}60 \\
88\end{array}$ & $\begin{array}{r}4 \\
30\end{array}$ & $\begin{array}{l}\mathrm{sp} \\
\mathrm{dp}\end{array}$ & M57 \\
\hline Jan. 51951 & $\begin{array}{l}37.0 \mathrm{~N} \\
706 \mathrm{E}\end{array}$ & 002 & $\begin{array}{l}\mathrm{a}^{\prime \prime} \\
\mathrm{b}\end{array}$ & $\stackrel{\mathrm{N}}{\mathrm{S}}$ & $\begin{array}{ll}80 & W \\
80 & \mathrm{E}\end{array}$ & $\begin{array}{l}30 \\
60\end{array}$ & $\begin{array}{l}90 \\
90\end{array}$ & $\begin{array}{l}-t \\
-t\end{array}$ & G57 \\
\hline Jan. 61951 & $\begin{array}{l}36 \mathrm{6} \mathrm{N} \\
70.9 \mathrm{E}\end{array}$ & 003 & $a^{\prime \prime}$ & $\stackrel{\mathrm{N}}{\mathrm{S}}$ & $\begin{array}{l}35 \mathrm{~W} \\
37 \mathrm{~W}\end{array}$ & $\begin{array}{l}32 \\
78\end{array}$ & $\begin{array}{l}22 \\
60\end{array}$ & $\underset{d p}{s p}$ & G57 \\
\hline $\begin{array}{l}\text { Aug. } 131951 \\
18: 58\end{array}$ & $\begin{array}{l}42.0 \mathrm{~N} \\
33.0 \mathrm{E}\end{array}$ & 000 & $\begin{array}{l}\mathrm{a}^{\prime \prime} \\
\mathrm{b}\end{array}$ & $\stackrel{\mathrm{N}}{\mathrm{N}}$ & $\begin{array}{l}20 \mathrm{~W} \\
73 \mathrm{E}\end{array}$ & $\begin{array}{l}80 \\
76\end{array}$ & $\begin{array}{l}14 \\
10\end{array}$ & $\underset{s p}{d p}$ & K58 \\
\hline Oct. 41951 & $\begin{array}{lll}36 & 6 & \mathrm{~N} \\
70 & 5 & \mathrm{E}\end{array}$ & 003 & $\begin{array}{l}\mathrm{a}^{\prime \prime} \\
\mathrm{b}\end{array}$ & $\stackrel{N}{\mathrm{~S}}$ & $\begin{array}{r}0 \mathrm{~W} \\
40 \mathrm{~W}\end{array}$ & $\begin{array}{l}40 \\
54\end{array}$ & $\begin{array}{l}59 \\
66\end{array}$ & $\underset{\mathrm{dp}}{\mathrm{dp}}$ & G57 \\
\hline $\begin{array}{l}\text { Oct. } 201951 \\
23: 22\end{array}$ & $\begin{array}{l}43.0 \mathrm{~N} \\
325 \mathrm{E}\end{array}$ & 000 & $\mathbf{a}^{\prime \prime}$ & $\mathbf{s}$ & $79 \mathrm{E}$ & $\begin{array}{l}84 \\
18\end{array}$ & 73 & $\begin{array}{l}-p \\
-p\end{array}$ & K58 \\
\hline $\begin{array}{l}\text { Oct. } 211951 \\
\text { 00:14 }\end{array}$ & $\begin{array}{lll}35 & 0 & \mathrm{~N} \\
46 & 0 & \mathrm{E}\end{array}$ & 000 & $a^{\prime \prime}$ & $\mathbf{S}$ & $78 \mathrm{E}$ & $\begin{array}{l}84 \\
18\end{array}$ & 73 & $\begin{array}{l}-\mathrm{p} \\
-\mathrm{p}\end{array}$ & K58 \\
\hline
\end{tabular}


TABLE 1-Contrnued

\begin{tabular}{|c|c|c|c|c|c|c|c|c|c|}
\hline A & $B$ & $\mathrm{C}$ & D & & $E$ & $\mathrm{~F}$ & G & H & I \\
\hline $\begin{array}{l}\text { Oct. } 211951 \\
03: 10\end{array}$ & $\begin{array}{ll}38 & 0 \mathrm{~N} \\
47 & 8 \mathrm{E}\end{array}$ & 0.00 & $a^{\prime \prime}$ & & $21 \mathrm{E}$ & $\begin{array}{l}52 \\
44\end{array}$ & 66 & $\begin{array}{l}-p \\
-p\end{array}$ & K58 \\
\hline $\begin{array}{l}\text { Nov. } 21951 \\
21: 55\end{array}$ & $\begin{array}{l}430 \mathrm{~N} \\
457 \mathrm{E}\end{array}$ & 000 & $\mathrm{a}^{\prime \prime}$ & $\mathrm{s}$ & $17 \mathrm{~W}$ & $\begin{array}{l}80 \\
16\end{array}$ & 77 & $-t$ & $\mathrm{~K} 58$ \\
\hline $\begin{array}{l}\text { Dec. } 121951 \\
17: 55\end{array}$ & $\begin{array}{ll}39 & 0 \mathrm{~N} \\
45 & 0 \mathrm{E}\end{array}$ & 000 & $a^{\prime \prime}$ & $\mathbf{s}$ & $12 \mathrm{E}$ & $\begin{array}{l}34 \\
61\end{array}$ & 60 & $\begin{array}{l}-p \\
-p\end{array}$ & K58 \\
\hline $\begin{array}{l}\text { Jan. } 41952 \\
15: 01\end{array}$ & $\begin{array}{lll}40.1 & \mathrm{~N} \\
59 & 0 \mathrm{E}\end{array}$ & 000 & $\mathbf{a}^{\prime \prime}$ & $\mathbf{S}$ & $12 \mathrm{~W}$ & $\begin{array}{l}80 \\
14\end{array}$ & 80 & $\begin{array}{l}-p \\
-p\end{array}$ & K58 \\
\hline $\begin{array}{l}\text { Feb. } 111952 \\
07: 01: 05\end{array}$ & $\begin{array}{rl}5 & 5 \mathrm{~S} \\
109 & 8 \mathrm{E}\end{array}$ & 010 & $\begin{array}{l}\mathrm{a}^{\prime \prime} \\
\mathrm{b}\end{array}$ & $\begin{array}{l}\mathrm{N} \\
\mathrm{N}\end{array}$ & $\begin{array}{l}86 \mathrm{~W} \\
18 \mathrm{E}\end{array}$ & $\begin{array}{l}64 \\
64\end{array}$ & $\begin{array}{l}29 \\
29\end{array}$ & $\begin{array}{l}\text { st } \\
\mathrm{dt}\end{array}$ & R57 \\
\hline $\begin{array}{l}\text { Feb. } 141952 \\
03: 38: 15\end{array}$ & $\begin{array}{r}7.7 \mathrm{~S} \\
1265 \mathrm{E}\end{array}$ & 000 & $\begin{array}{l}a^{\prime \prime} \\
b\end{array}$ & $\stackrel{N}{N}$ & $\begin{array}{l}27 \mathrm{E} \\
54 \mathrm{~W}\end{array}$ & $\begin{array}{l}87 \\
74\end{array}$ & $\begin{array}{r}37 \\
9\end{array}$ & $\begin{array}{l}\mathrm{dt} \\
\text { st }\end{array}$ & R57 \\
\hline $\begin{array}{l}\text { Mar. } 191952 \\
\text { 10:57:09 }\end{array}$ & $\begin{array}{r}9.5 \mathrm{~N} \\
126.0 \mathrm{E}\end{array}$ & 0.00 & $\begin{array}{l}\mathrm{a}^{\prime \prime} \\
\mathrm{b}\end{array}$ & $\stackrel{N}{N}$ & $\begin{array}{l}48 \mathrm{E} \\
78 \mathrm{~W}\end{array}$ & $\begin{array}{l}53 \\
52\end{array}$ & $\begin{array}{l}50 \\
50\end{array}$ & $\begin{array}{l}\mathrm{dt} \\
\text { st }\end{array}$ & R57 \\
\hline $\begin{array}{l}\text { Apr. } 181952 \\
05: 26\end{array}$ & $\begin{array}{ll}39 & 0 \mathrm{~N} \\
45 & 0 \mathrm{E}\end{array}$ & 000 & $\begin{array}{l}a^{\prime \prime} \\
b^{\prime \prime}\end{array}$ & $\mathrm{s}$ & $62 \mathrm{~W}$ & $\begin{array}{l}29 \\
70\end{array}$ & 45 & $\begin{array}{l}-p \\
-p\end{array}$ & K58 \\
\hline May 281952 & $\begin{array}{lll}37 & 0 \mathrm{~N} \\
70 & 8 \mathrm{E}\end{array}$ & 003 & $\begin{array}{l}a^{\prime \prime} \\
b^{\prime \prime}\end{array}$ & $\stackrel{\mathrm{S}}{\mathrm{S}}$ & $\begin{array}{l}60 \mathrm{~W} \\
55 \mathrm{E}\end{array}$ & $\begin{array}{l}20 \\
82\end{array}$ & $\begin{array}{l}26 \\
72\end{array}$ & $\begin{array}{l}\mathrm{sp} \\
\mathrm{dp}\end{array}$ & G57 \\
\hline July 51952 & $\begin{array}{ll}36 & 9 \mathrm{~N} \\
71 & 0 \mathrm{E}\end{array}$ & 0.03 & $\begin{array}{l}a^{\prime \prime} \\
b\end{array}$ & $\stackrel{N}{\mathrm{~N}}$ & $\begin{array}{l}46 \mathrm{E} \\
46 \mathrm{~W}\end{array}$ & $\begin{array}{l}10 \\
80\end{array}$ & $\begin{array}{l}90 \\
90\end{array}$ & $\begin{array}{l}-p \\
-p\end{array}$ & G57 \\
\hline $\begin{array}{l}\text { July } 131952 \\
17: 34: 30\end{array}$ & $\begin{array}{r}3.1 \mathrm{~S} \\
127.4 \mathrm{E}\end{array}$ & 0.00 & $\begin{array}{l}a^{\prime \prime} \\
b\end{array}$ & $\stackrel{N}{N}$ & $\begin{array}{l}59 \mathrm{~W} \\
73 \mathrm{E}\end{array}$ & $\begin{array}{l}26 \\
72\end{array}$ & $\begin{array}{l}45 \\
71\end{array}$ & $\begin{array}{l}\mathrm{dp} \\
\mathrm{sp}\end{array}$ & R57 \\
\hline $\begin{array}{l}\text { Jan. } 201953 \\
17: 33: 07\end{array}$ & $126 \stackrel{1.5 \mathrm{~N}}{\mathrm{E}}$ & 000 & $\begin{array}{l}a^{\prime \prime} \\
b\end{array}$ & $\stackrel{\mathrm{S}}{\mathrm{N}}$ & $\begin{array}{l}65 \mathrm{~W} \\
25 \mathrm{~W}\end{array}$ & $\begin{array}{l}80 \\
89\end{array}$ & $\begin{array}{r}1 \\
10\end{array}$ & $\begin{array}{l}\mathrm{sp} \\
\mathrm{dp}\end{array}$ & R57 \\
\hline $\begin{array}{l}\text { Apr. } 91953 \\
00: 36: 16\end{array}$ & $\begin{array}{r}73 \mathrm{~S} \\
131.0 \mathrm{E}\end{array}$ & 0.00 & $\begin{array}{l}\mathrm{a}^{\prime \prime} \\
\mathrm{b}\end{array}$ & $\stackrel{\mathrm{N}}{\mathrm{N}}$ & $68 \mathrm{~W}$ & $\begin{array}{l}59 \\
67\end{array}$ & $\begin{array}{l}27 \\
34\end{array}$ & $\begin{array}{l}\mathrm{dt} \\
\text { st }\end{array}$ & $\mathrm{R} 57$ \\
\hline $\begin{array}{l}\text { June } 251953 \\
10: 44: 57\end{array}$ & $\begin{array}{r}8.5 \mathrm{~S} \\
1235 \mathrm{E}\end{array}$ & 000 & $\begin{array}{l}\mathrm{a}^{\prime \prime} \\
\mathrm{b}\end{array}$ & $\stackrel{N}{N}$ & $\begin{array}{l}66 \mathrm{~W} \\
24 \mathrm{E}\end{array}$ & $\begin{array}{l}87 \\
88\end{array}$ & $\begin{array}{l}2 \\
3\end{array}$ & $\mathrm{dp}_{\mathrm{sp}}$ & R57 \\
\hline $\begin{array}{l}\text { July } 71953 \\
04: 07: 48\end{array}$ & $\begin{array}{rr}1 & \mathrm{~N} \\
100 & \mathrm{E}\end{array}$ & 0.03 & $\begin{array}{l}a^{\prime \prime} \\
b\end{array}$ & $\stackrel{\mathrm{S}}{\mathrm{N}}$ & $\begin{array}{l}40 \mathrm{E} \\
85 \mathrm{~W}\end{array}$ & $\begin{array}{l}31 \\
62\end{array}$ & $\begin{array}{l}51 \\
69\end{array}$ & $\underset{\mathrm{sp}}{\mathrm{dp}}$ & $\mathrm{R} 57$ \\
\hline $\begin{array}{l}\text { Nov. } 131953 \\
16: 17: 05\end{array}$ & $\begin{array}{r}35 \mathrm{~F} \\
96\end{array}$ & 000 & $\begin{array}{l}a^{\prime \prime} \\
b\end{array}$ & $\underset{S}{S}$ & $\begin{array}{l}72 \mathrm{E} \\
18 \mathrm{~W}\end{array}$ & $\begin{array}{l}89 \\
88\end{array}$ & $\begin{array}{l}2 \\
1\end{array}$ & $\begin{array}{l}\mathrm{dp} \\
\mathrm{sp}\end{array}$ & R57 \\
\hline $\begin{array}{l}\text { Dec. } 21953 \\
04: 24: 51\end{array}$ & $\begin{array}{r}28 \mathrm{~S} \\
1415 \mathrm{E}\end{array}$ & 0.00 & $\begin{array}{l}a^{\prime \prime} \\
b\end{array}$ & $\begin{array}{l}\mathrm{N} \\
\mathrm{S}\end{array}$ & $\begin{array}{l}50 \mathrm{~W} \\
40 \mathrm{~W}\end{array}$ & $\begin{array}{l}89 \\
88\end{array}$ & $\begin{array}{l}2 \\
1\end{array}$ & $\begin{array}{l}\mathrm{sp} \\
\mathrm{dp}\end{array}$ & R57 \\
\hline $\begin{array}{l}\text { Dec. } 71953 \\
02: 05: 37\end{array}$ & $\begin{array}{l}22 \mathrm{~S} \\
68.5 \mathrm{~W}\end{array}$ & 001 & $\begin{array}{l}a \\
b\end{array}$ & $\stackrel{N}{\mathrm{~S}}$ & $24 \underset{58}{\mathrm{E}}$ & $\begin{array}{l}55 \\
79\end{array}$ & $\begin{array}{l}14 \\
36\end{array}$ & $\begin{array}{l}\text { st } \\
d t\end{array}$ & I 57 \\
\hline $\begin{array}{l}\text { Jan. } 11954 \\
13: 04: 19\end{array}$ & $\begin{array}{r}90 \mathrm{~S} \\
123.5 \mathrm{E}\end{array}$ & 001 & $\begin{array}{l}a^{\prime \prime} \\
b\end{array}$ & $\stackrel{N}{\mathrm{~S}}$ & $\begin{array}{ll}56 & \mathrm{E} \\
23 & \mathrm{E}\end{array}$ & $\begin{array}{l}64 \\
89\end{array}$ & $\begin{array}{l}11 \\
28\end{array}$ & $\begin{array}{l}\mathrm{dp} \\
\mathrm{sp}\end{array}$ & R57 \\
\hline $\begin{array}{l}\text { Jan. } 171954 \\
17: 39: 38\end{array}$ & $\begin{array}{ll}16 & 5 \mathrm{~S} \\
36 & \mathrm{E}\end{array}$ & 000 & $\begin{array}{l}a^{\prime \prime} \\
b\end{array}$ & $\stackrel{N}{N}$ & $\begin{array}{l}73 \mathrm{~W} \\
24 \mathrm{E}\end{array}$ & $\begin{array}{l}68 \\
74\end{array}$ & $\begin{array}{l}17 \\
23\end{array}$ & $\mathrm{dp}_{\mathrm{sp}}$ & DeB56 \\
\hline $\begin{array}{l}\text { Feb. } 191954 \\
\text { 19:07:48 }\end{array}$ & $\stackrel{30}{177.7 \stackrel{S}{W}}$ & 000 & $\begin{array}{l}\mathrm{a} \\
\mathrm{b}\end{array}$ & $\underset{N}{S}$ & $\begin{array}{l}60 \mathrm{E} \\
30 \mathrm{E}\end{array}$ & $\begin{array}{l}87 \\
80\end{array}$ & $\begin{array}{r}10 \\
3\end{array}$ & $\begin{array}{l}\text { sp } \\
\mathrm{dp}\end{array}$ & HC57 \\
\hline
\end{tabular}


TABLE 1-Continued

\begin{tabular}{|c|c|c|c|c|c|c|c|c|}
\hline$A$ & B & $\mathrm{C}$ & $\mathrm{D}$ & E & F & G & $\mathrm{H}$ & I \\
\hline $\begin{array}{l}\text { Feb. } 191954 \\
21: 34: 41\end{array}$ & $\begin{array}{l}125 \mathrm{~N} \\
875 \mathrm{~W}\end{array}$ & 000 & $\begin{array}{l}\mathrm{a} \\
\mathrm{b}\end{array}$ & $\begin{array}{lll}\mathrm{N} & 62 & \mathrm{~W} \\
\mathrm{~N} & 35 & \mathrm{E}\end{array}$ & $\begin{array}{l}55 \\
82\end{array}$ & $\begin{array}{l}11 \\
36\end{array}$ & $\begin{array}{l}\text { st } \\
\mathrm{dt}\end{array}$ & $\mathrm{HC57}$ \\
\hline $\begin{array}{l}\text { Feb. } 201954 \\
18: 35: 07\end{array}$ & $\begin{array}{r}69 \mathrm{~S} \\
1245 \mathrm{E}\end{array}$ & 009 & $a^{\prime \prime}$ & $\begin{array}{lll}\mathrm{S} & 20 & \mathrm{E} \\
\mathrm{S} & 89 & \mathrm{~W}\end{array}$ & $\begin{array}{l}28 \\
80\end{array}$ & $\begin{array}{l}21 \\
64\end{array}$ & $\frac{\mathrm{sp}}{\mathrm{dp}}$ & R57 \\
\hline $\begin{array}{l}\text { Mar. } 31954 \\
06: 02: 55\end{array}$ & $\begin{array}{r}55 \mathrm{~S} \\
1425 \mathrm{E}\end{array}$ & 000 & $\begin{array}{l}a^{\prime \prime} \\
b\end{array}$ & $\begin{array}{lll}\mathrm{S} & 78 & \mathrm{E} \\
\mathrm{N} & 11 & \mathrm{E}\end{array}$ & $\begin{array}{l}76 \\
85\end{array}$ & $\begin{array}{r}5 \\
15\end{array}$ & $\begin{array}{l}\mathrm{dt} \\
\mathrm{st}\end{array}$ & R57 \\
\hline $\begin{array}{l}\text { Apr. } 171954 \\
20: 10: 37\end{array}$ & $\begin{array}{rl}51 & 5 \\
179 & \mathrm{~W}\end{array}$ & 000 & $\begin{array}{l}a \\
b\end{array}$ & $\begin{array}{lrl}\mathrm{N} & 2 & \mathrm{~W} \\
\mathrm{~S} & 87 & \mathrm{~W}\end{array}$ & $\begin{array}{l}82 \\
84\end{array}$ & $\begin{array}{l}6 \\
8\end{array}$ & $\begin{array}{l}\mathrm{dt} \\
\mathrm{st}\end{array}$ & $\mathrm{HC57}$ \\
\hline $\begin{array}{l}\text { Apr. } 271954 \\
10: 06: 24\end{array}$ & $\begin{array}{rr}6 & \mathrm{~N} \\
82 & 5 \mathrm{~W}\end{array}$ & 000 & $\begin{array}{l}a \\
b\end{array}$ & $\begin{array}{lrl}\mathrm{S} & 84 & \mathrm{E} \\
\mathrm{N} & 5 & \mathrm{E}\end{array}$ & $\begin{array}{l}85 \\
76\end{array}$ & $\begin{array}{r}14 \\
5\end{array}$ & $\begin{array}{l}\mathrm{dt} \\
\mathrm{st}\end{array}$ & $\mathrm{HC} 57$ \\
\hline $\begin{array}{l}\text { Apr. } 291954 \\
10: 49: 27\end{array}$ & $\begin{array}{r}28 \\
113\end{array} \stackrel{\mathrm{W}}{\mathrm{W}}$ & 000 & $\begin{array}{l}a \\
b\end{array}$ & $\begin{array}{lll}\mathrm{S} & 44 & \mathrm{E} \\
\mathrm{N} & 45 & \mathrm{E}\end{array}$ & $\begin{array}{l}88 \\
68\end{array}$ & $\begin{array}{r}22 \\
2\end{array}$ & $\begin{array}{l}\mathrm{sp} \\
\mathrm{dp}\end{array}$ & HC57 \\
\hline $\begin{array}{l}\text { Apr. } 301954 \\
13: 02: 37\end{array}$ & $\begin{array}{ll}39 & \mathrm{~N} \\
22 & \mathrm{E}\end{array}$ & 000 & $\begin{array}{l}a \\
b\end{array}$ & $\begin{array}{lrl}\mathrm{N} & 4 & \mathrm{~W} \\
\mathrm{~S} & 44 & \mathrm{~W}\end{array}$ & $\begin{array}{l}18 \\
78\end{array}$ & $\begin{array}{l}43 \\
77\end{array}$ & $\begin{array}{l}d t \\
\text { st }\end{array}$ & $\mathrm{HC} 57$ \\
\hline $\begin{array}{l}\text { May } 31954 \\
15: 29: 40\end{array}$ & $\begin{array}{r}515 \mathrm{~N} \\
1595 \mathrm{E}\end{array}$ & 000 & $\begin{array}{l}\mathrm{a} \\
\mathrm{b}\end{array}$ & $\begin{array}{lll}\mathrm{N} & 81 & \mathrm{~W} \\
\mathrm{~N} & 16 & \mathrm{E}\end{array}$ & $\begin{array}{l}70 \\
71\end{array}$ & $\begin{array}{l}20 \\
21\end{array}$ & $\begin{array}{l}\text { st } \\
\mathrm{dt}\end{array}$ & $\mathrm{HC} 57$ \\
\hline $\begin{array}{l}\text { May } 141954 \\
22: 39: 26\end{array}$ & $\begin{array}{rl}36 & \mathrm{~N} \\
137 & \mathrm{E}\end{array}$ & 003 & $\begin{array}{l}\mathrm{a} \\
\mathrm{b}\end{array}$ & $\begin{array}{lll}\mathrm{N} & 34 & \mathrm{~W} \\
\mathrm{~N} & 59 & \mathrm{E}\end{array}$ & $\begin{array}{l}68 \\
83\end{array}$ & $\begin{array}{r}8 \\
22\end{array}$ & $\begin{array}{l}\mathrm{dp} \\
\mathrm{sp}\end{array}$ & $\mathrm{HC} 57$ \\
\hline $\begin{array}{l}\text { June } 61954 \\
16: 50: 40\end{array}$ & $135.5 \mathrm{~S}$ & 000 & $\begin{array}{l}\mathrm{a}^{\prime \prime} \\
\mathrm{b}\end{array}$ & $\begin{array}{lll}\mathrm{N} & 64 & \mathrm{E} \\
\mathrm{S} & 14 & \mathrm{E}\end{array}$ & $\begin{array}{l}88 \\
15\end{array}$ & $\begin{array}{l}75 \\
12\end{array}$ & $\begin{array}{l}\mathrm{dt} \\
\mathrm{st}\end{array}$ & R57 \\
\hline $\begin{array}{l}\text { July } 31954 \\
00: 32: 57\end{array}$ & $\begin{array}{r}34 \mathrm{~S} \\
29.1 \mathrm{E}\end{array}$ & 000 & $\begin{array}{l}a^{\prime \prime} \\
b\end{array}$ & $\begin{array}{lll}\mathrm{S} & 81 & \mathrm{E} \\
\mathrm{N} & 11 & \mathrm{~W}\end{array}$ & $\begin{array}{l}58 \\
62\end{array}$ & $\begin{array}{l}34 \\
37\end{array}$ & $\begin{array}{l}\mathrm{dt} \\
\mathrm{st}\end{array}$ & DeB56 \\
\hline $\begin{array}{l}\text { July } 3 \quad 1954 \\
22: 31: 25\end{array}$ & $\begin{array}{r}65 \mathrm{~S} \\
105.5 \mathrm{E}\end{array}$ & 0.01 & $\begin{array}{l}a^{\prime \prime} \\
b\end{array}$ & $\begin{array}{lll}\mathbf{S} & 37 & \mathrm{~W} \\
\mathbf{N} & 15 & \mathrm{~W}\end{array}$ & $\begin{array}{l}66 \\
36\end{array}$ & $\begin{array}{l}62 \\
44\end{array}$ & dp & R57 \\
\hline $\begin{array}{l}\text { Aug. } 181954 \\
04: 42: 20\end{array}$ & $\begin{array}{rl}21 & 5 \mathrm{~S} \\
176 & \mathrm{~W}\end{array}$ & 0.02 & $\mathrm{a}$ & $\begin{array}{lll}\mathrm{S} & 63 & \mathrm{E} \\
\mathrm{N} & 23 & \mathrm{E}\end{array}$ & $\begin{array}{l}81 \\
69\end{array}$ & $\begin{array}{r}21 \\
9\end{array}$ & $\begin{array}{l}\mathrm{dt} \\
\mathrm{st}\end{array}$ & $\mathrm{HC} 57$ \\
\hline $\begin{array}{l}\text { Sept. } 131954 \\
02: 09: 55\end{array}$ & $\begin{array}{r}21 \\
175\end{array} 5 \stackrel{S}{W}$ & 0.02 & $\begin{array}{l}\mathrm{a} \\
\mathrm{b}\end{array}$ & $\begin{array}{lll}\mathrm{S} & 73 & \mathrm{E} \\
\mathrm{N} & 15 & \mathrm{E}\end{array}$ & $\begin{array}{l}84 \\
70\end{array}$ & $\begin{array}{r}20 \\
6\end{array}$ & $\begin{array}{l}d t \\
s t\end{array}$ & $\mathrm{HC} 57$ \\
\hline $\begin{array}{l}\text { Sept. } 151954 \\
17: 56: 08\end{array}$ & $\underset{178}{18} \underset{5}{\mathrm{~S}}$ & 0.09 & $\begin{array}{l}\mathrm{a} \\
\mathrm{b}\end{array}$ & $\begin{array}{llll}\mathrm{N} & 38 & \mathrm{~W} \\
\mathrm{~N} & 56 & \mathrm{E}\end{array}$ & $\begin{array}{l}83 \\
60\end{array}$ & $\begin{array}{r}30 \\
8\end{array}$ & $\begin{array}{l}\text { st } \\
\mathrm{dt}\end{array}$ & $\mathrm{HC} 57$ \\
\hline $\begin{array}{l}\text { Sept. } 201954 \\
00: 39: 28\end{array}$ & $\begin{array}{rl}1 & 5 \mathrm{~S} \\
120 & 5 \mathrm{E}\end{array}$ & 0.00 & $\begin{array}{l}a^{\prime \prime} \\
b\end{array}$ & $\begin{array}{lll}\mathrm{N} & 35 & \mathrm{~W} \\
\mathrm{~N} & 74 & \mathrm{E}\end{array}$ & $\begin{array}{l}38 \\
76\end{array}$ & $\begin{array}{l}23 \\
54\end{array}$ & $\underset{\mathrm{sp}}{\mathrm{dp}}$ & $\mathrm{R} 57$ \\
\hline $\begin{array}{l}\text { Oet. } 31954 \\
11: 18: 46\end{array}$ & 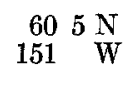 & 001 & $\begin{array}{l}\mathrm{a} \\
\mathrm{b}\end{array}$ & $\begin{array}{lll}\mathrm{S} & 54 & \mathrm{E} \\
\mathrm{S} & 44 & \mathrm{~W}\end{array}$ & $\begin{array}{l}52 \\
77\end{array}$ & $\begin{array}{l}15 \\
39\end{array}$ & $\begin{array}{l}\text { st } \\
\mathrm{dt}\end{array}$ & HC57 \\
\hline $\begin{array}{l}\text { Oct. } 31954 \\
23: 21: 35\end{array}$ & $\begin{array}{r}15 \mathrm{~S} \\
127.5 \mathrm{E}\end{array}$ & 000 & $\begin{array}{l}\mathrm{a}^{\prime \prime} \\
\mathrm{b}\end{array}$ & $\begin{array}{lll}\mathbf{N} & 21 & \mathbf{E} \\
\mathrm{N} & 70 & \mathrm{~W}\end{array}$ & $\begin{array}{l}87 \\
80\end{array}$ & $\begin{array}{l}10 \\
32\end{array}$ & $\stackrel{s p}{d p}$ & R57 \\
\hline $\begin{array}{l}\text { Nov. } 21954 \\
08: 24: 10\end{array}$ & $\begin{array}{r}80 \mathrm{~S} \\
1190 \mathrm{E}\end{array}$ & 000 & $\begin{array}{l}a^{\prime \prime} \\
b\end{array}$ & $\begin{array}{lrl}\mathrm{N} & 89 & \mathrm{~W} \\
\mathrm{~N} & 2 & \mathrm{E}\end{array}$ & $\begin{array}{l}87 \\
74\end{array}$ & $\begin{array}{r}16 \\
3\end{array}$ & $\begin{array}{l}d t \\
s t\end{array}$ & R57 \\
\hline $\begin{array}{l}\text { Dec. } 161954 \\
\text { 11:07:11 }\end{array}$ & $\begin{array}{r}393 \mathrm{~N} \\
118.1 \mathrm{~W}\end{array}$ & 0.00 & $\begin{array}{l}\mathrm{a} \\
\mathrm{b}\end{array}$ & $\begin{array}{lll}\mathrm{N} & 79 & \mathrm{E} \\
\mathrm{N} & 25 & \mathrm{~W}\end{array}$ & $\begin{array}{l}62 \\
66\end{array}$ & $\begin{array}{l}28 \\
31\end{array}$ & $\begin{array}{l}\mathrm{dt} \\
\mathrm{st}\end{array}$ & Ro57 \\
\hline $\begin{array}{l}\text { Jan. } 51955 \\
17: 48: 35\end{array}$ & $\begin{array}{c}16 \\
167.5 \mathrm{~S}\end{array}$ & 0.00 & $\begin{array}{l}\mathrm{a} \\
\mathrm{b}\end{array}$ & $\begin{array}{lll}\mathrm{S} & 34 & \mathrm{E} \\
\mathrm{N} & 56 & \mathrm{E}\end{array}$ & $\begin{array}{l}56 \\
89\end{array}$ & $\begin{array}{r}1 \\
34\end{array}$ & $\begin{array}{l}\mathrm{dt} \\
\mathrm{st}\end{array}$ & $\mathrm{HC} 57$ \\
\hline
\end{tabular}


TABLE 1-Continued

\begin{tabular}{|c|c|c|c|c|c|c|c|c|c|}
\hline A & B & C & $\mathbf{D}$ & & $\mathrm{E}$ & F & G & $\mathrm{H}$ & 1 \\
\hline $\begin{array}{l}\text { Jan. } 131955 \\
02: 03: 43\end{array}$ & $\begin{array}{rl}53 & \mathrm{~N} \\
167 & \mathrm{~W}\end{array}$ & 000 & $\begin{array}{l}a \\
b\end{array}$ & $\stackrel{\mathrm{S}}{\mathrm{N}}$ & $\begin{array}{ll}38 & \mathrm{E} \\
51 & \mathrm{E}\end{array}$ & $\begin{array}{l}89 \\
74\end{array}$ & $\begin{array}{r}16 \\
2\end{array}$ & $\begin{array}{l}\mathrm{sp} \\
\mathrm{dp}\end{array}$ & $\mathrm{HC} 57$ \\
\hline $\begin{array}{l}\text { Mar. } 141955 \\
13: 12: 04\end{array}$ & $\begin{array}{rll}52 & 5 & \mathrm{~N} \\
173 & 5 & \mathrm{~W}\end{array}$ & 001 & $\begin{array}{l}a \\
b\end{array}$ & $\stackrel{\mathrm{N}}{\mathrm{N}}$ & $\begin{array}{l}71 \mathrm{~W} \\
23 \mathrm{E}\end{array}$ & $\begin{array}{l}70 \\
79\end{array}$ & $\begin{array}{l}12 \\
20\end{array}$ & $\begin{array}{l}\text { st } \\
\mathrm{dt}\end{array}$ & $\mathrm{HC} 57$ \\
\hline $\begin{array}{l}\text { Apr. } 171955 \\
18: 35: 27\end{array}$ & $\begin{array}{rl}52 & \mathrm{~N} \\
159 & \mathrm{E}\end{array}$ & 000 & $\begin{array}{l}\mathrm{a} \\
\mathrm{b}\end{array}$ & $\begin{array}{l}\mathrm{N} \\
\mathrm{N}\end{array}$ & $\begin{array}{l}39 \mathrm{~W} \\
52 \mathrm{E}\end{array}$ & $\begin{array}{l}84 \\
78\end{array}$ & $\begin{array}{r}12 \\
6\end{array}$ & $\begin{array}{l}\text { st } \\
\text { dt }\end{array}$ & HC57 \\
\hline $\begin{array}{l}\text { Apr. } 191955 \\
20: 24: 05\end{array}$ & $\begin{array}{ll}30 & \mathrm{~S} \\
72 & \mathrm{~W}\end{array}$ & 000 & $\begin{array}{l}a \\
b\end{array}$ & $\mathrm{~N}$ & $\begin{array}{l}55 \mathrm{~W} \\
41 \mathrm{E}\end{array}$ & $\begin{array}{l}72 \\
72\end{array}$ & $\begin{array}{l}19 \\
19\end{array}$ & $\begin{array}{l}\text { st } \\
d t\end{array}$ & HC57 \\
\hline $\begin{array}{l}\text { Mav } 301955 \\
12: 31: 41\end{array}$ & $\begin{array}{r}245 \mathrm{~N} \\
1425 \mathrm{E}\end{array}$ & 009 & $\begin{array}{l}\mathrm{a} \\
\mathrm{b}\end{array}$ & $\begin{array}{l}\mathrm{N} \\
\mathrm{S}\end{array}$ & $\begin{array}{l}20 \mathrm{~W} \\
70 \mathrm{~W}\end{array}$ & $\begin{array}{l}35 \\
90\end{array}$ & $\begin{array}{r}0 \\
55\end{array}$ & $\begin{array}{l}\mathrm{dt} \\
\mathrm{st}\end{array}$ & HC57 \\
\hline $\begin{array}{l}\text { June } 201955 \\
12: 07: 25\end{array}$ & $\begin{array}{r}515 \mathrm{~N} \\
180\end{array}$ & 000 & $\begin{array}{l}\mathrm{a} \\
\mathrm{b}\end{array}$ & $\begin{array}{l}\mathrm{N} \\
\mathrm{N}\end{array}$ & $\begin{array}{l}52 \mathrm{~W} \\
63 \mathrm{E}\end{array}$ & $\begin{array}{l}58 \\
56\end{array}$ & $\begin{array}{l}41 \\
40\end{array}$ & $\begin{array}{l}\text { st } \\
d t\end{array}$ & $\mathrm{HC} 57$ \\
\hline $\begin{array}{l}\text { July } 161955 \\
07: 07: 08\end{array}$ & $\begin{array}{ll}37 & 5 \mathrm{~N} \\
27 & \mathrm{E}\end{array}$ & 000 & $\begin{array}{l}\mathrm{a} \\
\mathrm{b}\end{array}$ & $\underset{N}{N}$ & $\begin{array}{l}50 \mathrm{~W} \\
40 \mathrm{E}\end{array}$ & $\begin{array}{l}84 \\
84\end{array}$ & $\begin{array}{l}6 \\
6\end{array}$ & $\underset{s p}{d p}$ & $\mathrm{HC} 57$ \\
\hline $\begin{array}{l}\text { Aug. } 161955 \\
11: 46: 58\end{array}$ & $\begin{array}{rl}6 & \mathrm{~S} \\
155 & \mathrm{E}\end{array}$ & 003 & $\mathrm{a}$ & $\frac{\mathrm{N}}{\mathrm{N}}$ & $\begin{array}{l}41 \mathrm{~W} \\
52 \mathrm{E}\end{array}$ & $\begin{array}{l}81 \\
71\end{array}$ & $\begin{array}{r}19 \\
9\end{array}$ & $\begin{array}{l}d p \\
s p\end{array}$ & HS58 \\
\hline $\begin{array}{l}\text { Aug. } 211955 \\
17: 33: 58\end{array}$ & $\begin{array}{rr}3 & \mathrm{~S} \\
137 & \mathrm{E}\end{array}$ & 0.00 & $\mathrm{a}$ & $\stackrel{S}{N}$ & $\begin{array}{l}16 \mathrm{E} \\
64 \mathrm{E}\end{array}$ & $\begin{array}{l}60 \\
72\end{array}$ & $\begin{array}{l}21 \\
31\end{array}$ & $\frac{\mathrm{sp}}{\mathrm{dp}}$ & HS58 \\
\hline $\begin{array}{l}\text { Aug. } 281955 \\
20: 13: 30\end{array}$ & $\begin{array}{ll}14 & \mathrm{~N} \\
91 & \mathrm{~W}\end{array}$ & 001 & $\begin{array}{l}a \\
b\end{array}$ & $\underset{\mathrm{N}}{\mathrm{S}}$ & $83 \mathrm{E}$ & $\begin{array}{l}73 \\
54\end{array}$ & $\begin{array}{l}38 \\
21\end{array}$ & $\begin{array}{l}\mathrm{sp} \\
\mathrm{dp}\end{array}$ & HS58 \\
\hline $\begin{array}{l}\text { Sept. } 121955 \\
06: 09: 20\end{array}$ & $\begin{array}{ll}32 & 5 \mathrm{~N} \\
30 & \mathrm{E}\end{array}$ & 000 & $\begin{array}{l}\mathrm{a} \\
\mathrm{b}\end{array}$ & $\begin{array}{l}\mathrm{N} \\
\mathrm{N}\end{array}$ & $\begin{array}{l}57 \mathrm{~W} \\
52 \mathrm{E}\end{array}$ & $\begin{array}{l}64 \\
56\end{array}$ & $\begin{array}{l}39 \\
32\end{array}$ & $\begin{array}{l}\mathrm{dp} \\
\mathrm{sp}\end{array}$ & HS58 \\
\hline $\begin{array}{l}\text { Nov. } 221955 \\
03: 24: 00\end{array}$ & $\begin{array}{rl}24 & 5 \\
123 & \mathrm{~S} \\
\mathrm{~W}\end{array}$ & 000 & $\begin{array}{l}\mathrm{a} \\
\mathrm{b}\end{array}$ & $\stackrel{N}{N}$ & $\begin{array}{l}88 \mathrm{~W} \\
02 \mathrm{E}\end{array}$ & $\begin{array}{l}89 \\
79\end{array}$ & $\begin{array}{r}11 \\
1\end{array}$ & $\underset{\mathrm{sp}}{\mathrm{dp}}$ & HS58 \\
\hline $\begin{array}{l}\text { Jan. } 81956 \\
20: 54: 13\end{array}$ & $\begin{array}{ll}19 & \mathrm{~S} \\
70 & \mathrm{~W}\end{array}$ & 000 & $\begin{array}{l}a \\
b\end{array}$ & $\frac{\mathrm{N}}{\mathrm{N}}$ & $\begin{array}{ll}72 & W \\
21 & \mathrm{E}\end{array}$ & $\begin{array}{l}83 \\
67\end{array}$ & $\begin{array}{r}23 \\
8\end{array}$ & $\begin{array}{l}\text { st } \\
\mathrm{dt}\end{array}$ & HS58 \\
\hline $\begin{array}{l}\text { Jan. } 101956 \\
08: 52: 36\end{array}$ & $\begin{array}{rl}25 & \mathrm{~S} \\
176 & \mathrm{~W}\end{array}$ & 000 & $\begin{array}{l}\mathrm{a} \\
\mathrm{b}\end{array}$ & $\stackrel{\mathrm{S}}{\mathrm{N}}$ & $\begin{array}{l}65 \mathrm{E} \\
23 \mathrm{E}\end{array}$ & $\begin{array}{l}82 \\
79\end{array}$ & $\begin{array}{r}11 \\
8\end{array}$ & $\begin{array}{l}\mathrm{dt} \\
\text { st }\end{array}$ & HS58 \\
\hline $\begin{array}{l}\text { Feb. } 91956 \\
14: 32: 40\end{array}$ & $\begin{array}{rl}31 & 5 \mathrm{~N} \\
116 & \mathrm{~W}\end{array}$ & 000 & $\begin{array}{l}\mathrm{a} \\
\mathrm{b}\end{array}$ & $\stackrel{\mathbf{S}}{\mathbf{N}}$ & $\begin{array}{ll}71 & \mathrm{E} \\
18 & \mathrm{E}\end{array}$ & $\begin{array}{l}85 \\
72\end{array}$ & $\begin{array}{r}18 \\
5\end{array}$ & $\mathrm{sp}$ & HS58 \\
\hline $\begin{array}{l}\text { Feb. } 181956 \\
07: 34: 16\end{array}$ & $\begin{array}{cc}30 & \mathrm{~N} \\
137 & 5 \mathrm{E}\end{array}$ & 007 & $\begin{array}{l}\mathrm{a} \\
\mathrm{b}\end{array}$ & $\frac{\mathrm{S}}{\mathrm{N}}$ & $\begin{array}{l}61 \mathrm{E} \\
14 \mathrm{E}\end{array}$ & $\begin{array}{l}56 \\
68\end{array}$ & $\begin{array}{l}27 \\
37\end{array}$ & $\begin{array}{l}\mathrm{dt} \\
\mathrm{st}\end{array}$ & HS58 \\
\hline $\begin{array}{l}\text { July } 91956 \\
03: 11: 39\end{array}$ & $\begin{array}{ll}37 & \mathrm{~N} \\
26 & \mathrm{E}\end{array}$ & 000 & $\begin{array}{l}\mathrm{a} \\
\mathrm{b}\end{array}$ & $\begin{array}{l}\mathrm{N} \\
\mathrm{S}\end{array}$ & $\begin{array}{l}47 \underset{W}{W} \\
28\end{array}$ & $\begin{array}{l}72 \\
55\end{array}$ & $\begin{array}{l}37 \\
22\end{array}$ & $\begin{array}{l}\mathrm{dt} \\
\mathrm{st}\end{array}$ & HS58 \\
\hline $\begin{array}{l}\text { July } 91956 \\
09: 56: 13\end{array}$ & $\begin{array}{ll}20 & \mathrm{~N} \\
73 & \mathrm{~W}\end{array}$ & 001 & $\begin{array}{l}a \\
b\end{array}$ & $\stackrel{\mathrm{S}}{\mathrm{N}}$ & $\begin{array}{r}77 \mathrm{E} \\
8 \mathrm{E}\end{array}$ & $\begin{array}{l}64 \\
79\end{array}$ & $\begin{array}{l}12 \\
27\end{array}$ & $\mathrm{sp}$ & HS58 \\
\hline
\end{tabular}




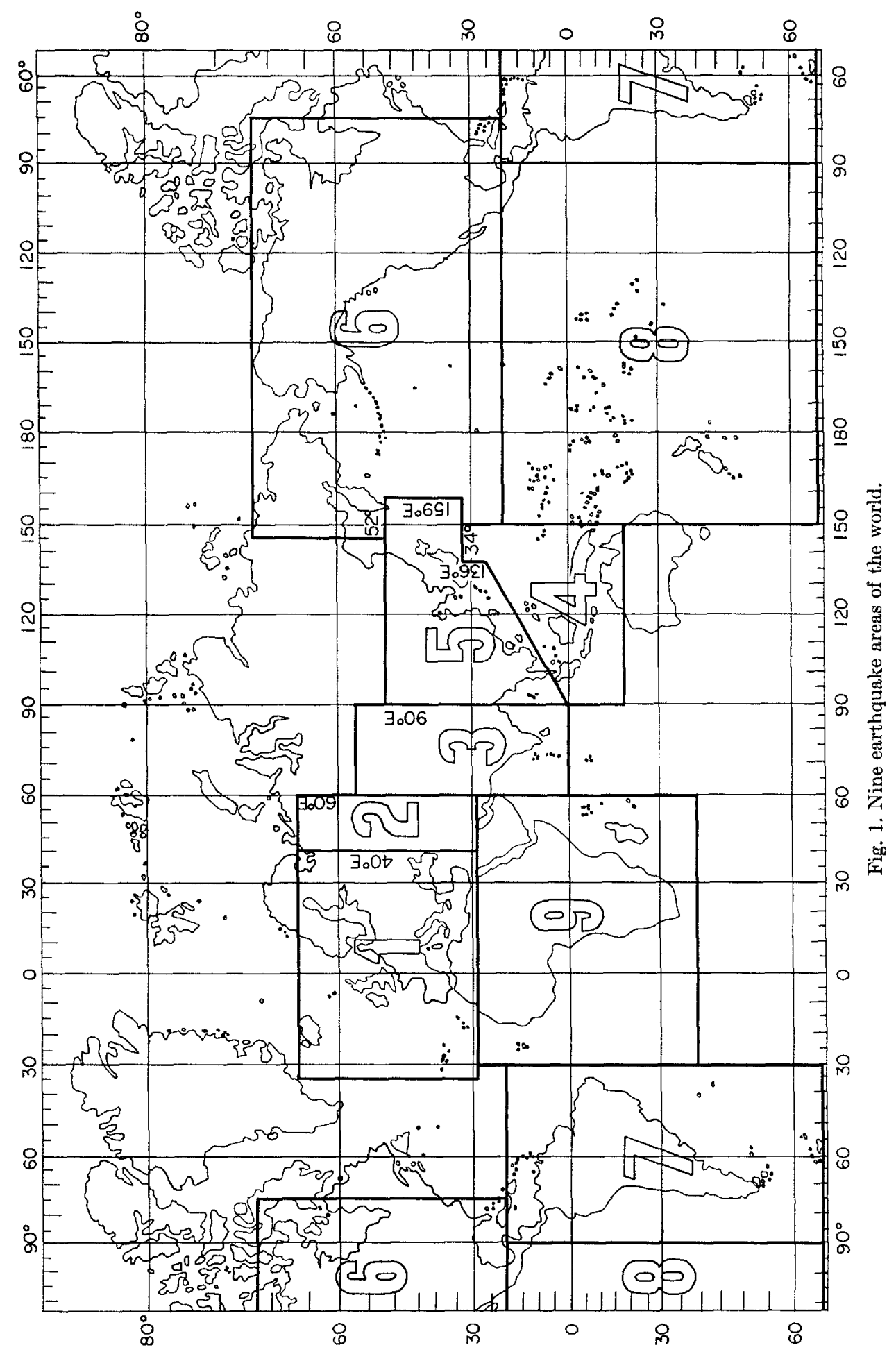


It has therefore been decided to recalculate the earlier tables of averages, using only "teleseisms." If this be done, only 164 of the earthquakes previously collected by the writer (Scheidegger, 1955, 1957 $a, 1957 b$ ) are usable. To these are now added 101 new fault-plane solutions (to make a total of 265), extracted from a variety of recent references. As in our earlier work, these solutions have been tabulated in a standard fashion and are shown in table 1 . Thus, we list the date and time (column A) and the location of the epicenters (column B) of the shocks as given by the individual authors, the depth in $0.0 \mathrm{n} \mathrm{R}$ (column $\mathrm{C}$ ), and the two planes, one of which represents the fault plane, the other the auxiliary plane. These two planes are given by their dip (column F) and dip direction (column E). If it be known which of the two planes represents the fault plane, that solution is marked by two primes (column D). Finally, the "slip angle," i.e., the angle between the strike direction of the fault plane and the direction of the motion axis, has been calculated for each fault-plane solution (column $G$ ). The character of the fault is shown in column $\mathrm{H}$ (s for sinistral, $\mathrm{d}$ for dextral, $\mathrm{t}$ for tensional, $\mathrm{p}$ for compressional) and the literature reference is given in column $\mathrm{I}$.

\section{Statistical Analysis}

As in our earlier papers, the world has been dissected into large earthquake areas, of which there are now nine (see fig. 1). With regard to these areas various averages have been calculated from the fault-plane solutions, in conformity with the earlier procedures. For the statistical averages each earthquake has been given double weight, so that if it was not clear which was the fault plane and which the auxiliary plane, both possibilities could be counted. If it was known which was the fault plane, then only that solution has been used, but then it has been given double weight.

The result of the calculations is shown in tables 2 to 6 . Earlier findings are thereby corroborated, which means (i) that, in the majority of cases, earthquake faulting is predominantly transcurrent; and (ii) that there are exceptions to the general transcurrency of faulting in the areas 2 (Caspian Sea), 3 (Pamir Knot and Hindu Kush), and possibly 4 (Indonesia).

However, the number of fault-plane solutions has now so much increased that it becomes possible to draw further inferences. It is feasible to make a breakdown with regard to depth (see table 3 ). If this be done, a very peculiar situation occurs at $0.03 \mathrm{R}$ : it is seen that the ratio of the number of tension to pressure earthquakes as well as the ratio of the number of strike slip to dip slip earthquakes becomes a minimum. The slip angle, consequently, has a maximum at that depth. This is very peculiar, but it is perhaps too early to attach too much significance to the observation. A check of the earthquakes reveals that a good number of them (17) happen to lie in the areas 3 and 4 which are known to be exceptional. Thus we may be faced with a regional peculiarity rather than with one that is conditioned by the depth.

Another investigation that might be of interest is one with regard to the effect of depth in the various areas. In conformity with the idea that there might be an orogenetic decoupling layer at $140 \mathrm{~km}$. depth (Gutenberg, 1955; Press, 1959), we term the earthquakes up to and including a depth of $0.02 \mathrm{R}$ as "shallow," the others as "deep." This is not in conformity with the common usage of the words, but it seems to be adequate for orogenetic considerations. 
TABLE 2

ALl EArThQUakes

\begin{tabular}{|c|c|c|c|c|c|c|c|c|c|c|}
\hline \multirow{2}{*}{ Region } & \multirow{2}{*}{$\begin{array}{l}\text { No. of } \\
\text { earth- } \\
\text { quakes }\end{array}$} & \multicolumn{3}{|c|}{ Per cent } & \multirow{2}{*}{$\begin{array}{l}\text { Ratio ten. } \\
\text { to pres. }\end{array}$} & \multicolumn{3}{|c|}{ Per cent } & \multirow{2}{*}{$\begin{array}{l}\text { Ratio st sl } \\
\text { to dap sl. }\end{array}$} & \multirow{2}{*}{$\begin{array}{c}\text { Avg } \\
\text { slp } \\
\text { angle }\end{array}$} \\
\hline & & Pres. & Ten. & Ind. & & Strike slpp & Dip slip & Ind. & & \\
\hline 1 & 17 & 17.6 & 52.9 & 29.4 & 3.000 & 88.2 & 11.8 & 0.0 & 7.500 & 23 \\
\hline 2 & 12 & 58.3 & 41.7 & 00 & 0.714 & 125 & 87.5 & 0.0 & 0.143 & 67 \\
\hline 3 & 14 & 64.3 & 28.6 & 7.1 & 0.444 & 28.6 & 60.7 & 10.7 & 0.470 & 64 \\
\hline 4 & 59 & 40.7 & 45.8 & 13.6 & 1.125 & 47.4 & 50.8 & 1.7 & 0.933 & 45 \\
\hline 5 & 73 & 27.4 & 16.4 & $\begin{array}{ll}562 & 2\end{array}$ & 0600 & 753 & 22.6 & 2.1 & 3.333 & 26 \\
\hline 6 & 32 & 40.6 & 59.4 & 00 & 1.462 & 76.6 & 219 & 1.6 & 3500 & 30 \\
\hline 7 & 13 & 23.1 & 53.8 & 23.1 & 2333 & 92.3 & 38 & 3.8 & 24.000 & 19 \\
\hline 8 & 43 & 651 & 25.6 & 9.3 & 0.393 & 941 & 3.5 & 23 & 27000 & 14 \\
\hline 9 & 2 & 50.0 & 50.0 & 00 & 1000 & 100.0 & 0.0 & 00 & $\infty$ & 26 \\
\hline World & 265 & 40.8 & 35.8 & 23.4 & 0.880 & 68.8 & 28.9 & 2.3 & 2386 & 32 \\
\hline
\end{tabular}

TABLE 3

Distribution with Depth

\begin{tabular}{|c|c|c|c|c|c|c|c|c|c|c|}
\hline \multirow{2}{*}{ Depth } & \multirow{2}{*}{$\begin{array}{l}\text { No of } \\
\text { earth- } \\
\text { quakes }\end{array}$} & \multicolumn{3}{|c|}{ Per cent } & \multirow{2}{*}{$\begin{array}{l}\text { Ratio ten. } \\
\text { to pres. }\end{array}$} & \multicolumn{3}{|c|}{ Per cent } & \multirow{2}{*}{$\begin{array}{l}\text { Ratro st. sl. } \\
\text { to dıp sl. }\end{array}$} & \multirow{2}{*}{$\begin{array}{l}\text { Avg. } \\
\text { slpp } \\
\text { angle }\end{array}$} \\
\hline & & Pres. & Ten. & Ind. & & Strike slip & Dip slip & Ind & & \\
\hline $0.00 \mathrm{R}$ & 143 & 37.7 & 37.7 & 245 & 1.000 & 787 & 20.6 & 0.7 & 3.814 & 25 \\
\hline $0.01 \mathbf{R}$ & 27 & 556 & 222 & 222 & 0400 & 63.0 & 315 & 5.6 & 2.000 & 35 \\
\hline $0.02 \mathrm{R}$ & 9 & 444 & 44.4 & 111 & 1.000 & 667 & 333 & 0.0 & 2.000 & 38 \\
\hline $0.03 \mathrm{R}$ & 26 & 61.5 & 270 & 11.5 & 0.438 & 34.6 & 558 & 9.6 & 0.621 & 55 \\
\hline $0.04 \mathbf{R}$ & 6 & 500 & 33.3 & 167 & 0667 & 91.7 & 83 & 0.0 & 11000 & 21 \\
\hline $0.05 \mathrm{R}$ & 20 & 350 & 200 & 450 & 0.571 & 62.5 & 325 & 5.0 & 1923 & 39 \\
\hline $0.06 \mathrm{R}$ & 4 & 250 & 25.0 & 50.0 & 1000 & 500 & 500 & 0.0 & 1000 & 36 \\
\hline $0.07 \mathrm{R}$ & 7 & 28.6 & 57.1 & 143 & 2.000 & 571 & 42.9 & 0.0 & 1.333 & 45 \\
\hline $0.08 \mathrm{R}$ & 7 & 286 & 714 & 00 & 2.500 & 50.0 & 500 & 0.0 & 1.000 & 50 \\
\hline $0.09 \mathrm{R}$ & 10 & 200 & 60.0 & 200 & 3000 & 60.0 & 40.0 & 0.0 & 1.500 & 37 \\
\hline $0.10 \mathrm{R}$ & 6 & 333 & 50.0 & 16.7 & 1.500 & 75.0 & 25.0 & 0.0 & 3.000 & 31 \\
\hline
\end{tabular}

TABLE 4

Shallow EarthQUakes

\begin{tabular}{|c|c|c|c|c|c|c|c|c|c|c|}
\hline \multirow{2}{*}{ Region } & \multirow{2}{*}{$\begin{array}{l}\text { No. of } \\
\text { earth- } \\
\text { quakes }\end{array}$} & \multicolumn{3}{|c|}{ Per cent } & \multirow{2}{*}{$\begin{array}{l}\text { Ratio ten. } \\
\text { to pres. }\end{array}$} & \multicolumn{3}{|c|}{ Per cent } & \multirow{2}{*}{$\begin{array}{l}\text { Rato st. sl. } \\
\text { to dip sl. }\end{array}$} & \multirow{2}{*}{$\begin{array}{c}\text { Avg } \\
\text { slup } \\
\text { angle }\end{array}$} \\
\hline & & Pres. & Ten. & Ind. & & Strike slp & Dip slip & Ind. & & \\
\hline 1 & 16 & 12.5 & 56.2 & 31.2 & 4500 & 90.6 & 9.4 & 00 & 9667 & 22 \\
\hline 2 & 12 & 583 & 41.7 & 0.0 & 0.714 & 12.5 & 87.5 & 00 & 0143 & 67 \\
\hline 3 & 5 & 20.0 & 60.0 & 20.0 & 3.000 & 400 & 500 & 10.0 & 0.800 & 55 \\
\hline 4 & 25 & 60.0 & 36.0 & 40 & 0600 & 660 & 34.0 & 0.0 & 1.941 & 32 \\
\hline 5 & 46 & 23.9 & 65 & 69.6 & 0.273 & 761 & 21.7 & 22 & 3500 & 22 \\
\hline 6 & 31 & 387 & 613 & 00 & 1583 & 790 & 194 & 16 & 4.083 & 29 \\
\hline 7 & 11 & 27.3 & 636 & 91 & 2333 & 909 & 45 & 4.5 & 20.000 & 21 \\
\hline 8 & 31 & 67.7 & 258 & 64 & 0381 & 952 & 48 & 00 & 19667 & 15 \\
\hline 9 & 2 & 500 & 500 & 0.0 & 1.000 & 100.0 & 00 & 0.0 & $\infty$ & 26 \\
\hline World & 179 & 408 & 35.8 & 23.5 & 0877 & 757 & 229 & 1.4 & 3.305 & 27 \\
\hline
\end{tabular}


TABLE 5

Deep Earthquakes

\begin{tabular}{|c|c|c|c|c|c|c|c|c|c|c|}
\hline \multirow{2}{*}{ Region } & \multirow{2}{*}{$\begin{array}{l}\text { No of } \\
\text { earth- } \\
\text { quakes }\end{array}$} & \multicolumn{3}{|c|}{ Per cent } & \multirow{2}{*}{$\begin{array}{l}\text { Ratio ten, } \\
\text { to pres }\end{array}$} & \multicolumn{3}{|c|}{ Per cent } & \multirow{2}{*}{$\begin{array}{c}\text { Ratio st sl sl. } \\
\text { to dip sl. }\end{array}$} & \multirow{2}{*}{$\begin{array}{l}\text { Avg } \\
\text { silp } \\
\text { angle }\end{array}$} \\
\hline & & Pres. & Ten. & Ind & & Strke slpp & Dip slp & Ind & & \\
\hline $\begin{array}{l}1 \\
2\end{array}$ & 1 & 1000 & 0.0 & 00 & 0000 & 500 & 500 & 00 & 1000 & 44 \\
\hline 3 & 9 & 88.9 & 111 & 00 & 0125 & 222 & 667 & 11.1 & 0.333 & 70 \\
\hline 4 & 34 & 265 & 529 & 206 & 2000 & 338 & 632 & 29 & 0535 & 55 \\
\hline 5 & 27 & 333 & 333 & 333 & 1000 & 741 & 241 & 18 & 3.077 & 40 \\
\hline 6 & 1 & 1000 & 00 & 00 & 0.000 & 00 & 1000 & 00 & 0.000 & 78 \\
\hline 7 & 2 & 00 & $\begin{array}{ll}0 & 0\end{array}$ & 1000 & & 1000 & 00 & 00 & $\infty$ & 5 \\
\hline 8 & 12 & 58.3 & 250 & 167 & 0428 & 917 & 0.0 & 83 & $\infty$ & 12 \\
\hline $\begin{array}{c}9 \\
\text { World }\end{array}$ & 86 & 407 & 36.0 & 23.2 & 0866 & 546 & 413 & 4.1 & $\ldots \ldots$ & 43 \\
\hline
\end{tabular}

TABLE 6

Comparison of Deep and Shallow Earthquakes

\begin{tabular}{|c|c|c|c|c|c|c|}
\hline \multirow{2}{*}{ Region } & \multicolumn{2}{|c|}{ Ratio of tension to pressure } & \multicolumn{2}{|c|}{ Ratio of strike slip to dup shp } & \multicolumn{2}{|c|}{ Slip angle } \\
\hline & Deep & Shallow & Deep & Shallow & Deep & Shallow \\
\hline 3. & 0125 & 3.000 & 0.333 & 0800 & 70 & 55 \\
\hline ... & 2000 & 0600 & 0.535 & 1.941 & 55 & 32 \\
\hline 5. . . . . . & 1000 & 0273 & 3077 & 3500 & 40 & 22 \\
\hline$\ldots \ldots \ldots$ & 0428 & 0.381 & $\infty$ & 19.667 & 12 & 15 \\
\hline World & 0886 & 0877 & 1. 324 & 3305 & 43 & 27 \\
\hline
\end{tabular}

The result of this investigation is shown in tables 4 and 5. A more direct collation of the results has been attempted in table 6 . It is only in regions $3,4,5$, and 8 that a proper comparison can be made, since only there do we have enough deep-focus earthquakes (in the sense used above) to permit our obtaining any significant results. An inspection of the last table (table 6) shows that there is a pretty good correlation" between the results from the "deep" and "shallow" earthquakes in any one area, as well as in the world as a whole. This seems to indicate that whatever it is that causes earthquakes acts in a similar fashion at small and at great depths. At any rate, there does not seem to be a definite break in the failure pattern at some intermediate depth. Unfortunately, the number of fault-plane solutions is still far too small to permit our making an analysis in terms of smaller depth steps (say: $0.01 \mathrm{R}$ ) for all the nine earthquake areas under consideration.

\section{CONCLUSION}

Reviewing the work reported above, it is seen that a statistical analysis of faultplane solutions provides a means for a discussion of geotectonics. Each individual fault-plane solution has little significance by itself, since the characteristics of the

1 The dip-slip components are somewhat erratic; the strike-slip components run exactly parallel in all areas, although they are, on the whole, somewhat smaller in shallow earthquakes. 
fault in any single earthquake seem due mostly to chance. However, averages that can be calculated for earthquake regions have a connection with the prevailing stress system. The various results obtained above will therefore have to be taken into account in any discussion of geotectonics.

\section{ACKNOWLEDGMENTS}

The present study was carried out at the California Institute of Technology in Pasadena, where the writer has been invited to spend a period as visitor. The writer is grateful to R. P. Sharp, Chairman of the Division of Geological Sciences, and F. Press, Director of the Seismological Laboratory, for the invitation and for the opportunity thus afforded to him to pursue his seismological research.

\section{REFERENCES}

[Symbols used in column I of table 1 are given in square brackets.]

De Bremaecker, J. C.

1956. Bull. Séances Acad. Roy. Sci. Col. Belg., 2: 762. [DeB 56]

Di Filippo, D., and L. Marcelli

1957. Annals Geofis. (Roma), 10: 221. [DM 57]

Gibowicz, S.

1957. Acta Geophys. Polon., 5: 202. [Gi 57]

Gotsadze, O. D., et al.

1957. "Issledovanie mekhanizma zemletryasenii, Trudy Geofiz. In-ta Akad. Nauk SSSR, No. 40 (166). 148 pp. [G 57]

Gutenberg, B.

1955. Bull. Geol. Soc. Am., 66: 1203.

Hodgson, J. H., and J. I. Cock

1957. Pub. Dom. Obs., Vol. 19, No. 6. [HC 57]

Hodgson, J. H., and A. Stevens

1958. Pub. Dom. Obs., Vol. 19, No. 8. [HS 58]

Ingram, R. E.

1957. Bull. Seism. Soc. Am., 47: 281. [I 57]

Karapetyan, N. K.

1958. Izv. Akad. Nauk SSSR, Ser. Geofiz., 1958:260. [K 58]

Mühlhảuser, $\mathbf{S}$.

1957. Tellus, 9: 104. [M 57]

Press, F.

1959. Jour. Geophys. Res., 64: 565.

Ritsema, A. R.

1957. Verh. Kem. Per. Lem. Met. Geof. Djakarta, No. 52. [R 57]

Romney, C.

1957. Bull. Seism. Soc. Am., 47: 301. [Ro 57]

Scheidegger, A. E.

1955. Trans. Roy. Soc. Canada, Sec. IV, 49: 65.

1957a. Geofis. Pura Appl., 38: 1.

1957b. Pub. Dom. Obs., Vol. 18, No. 3.

Veldkamp, $\mathbf{J}$.

1957. Verh. K. Ned. Mijnb. Gen. Geol. Ser., 18: 295. [V 57]

California Institute of Technology,

Pasadena, California.

(Division of Geological Sciences, contribution no. 926.) 